Maria Sepúlveda, MD

Thaís Armangué, MD

Nuria Sola-Valls, MD

Georgina Arrambide, MD

José E. Meca-Lallana, MD

Celia Oreja-Guevara, MD

Mar Mendibe, MD

Amaya Alvarez de Arcaya, MD

Yolanda Aladro, MD

Bonaventura Casanova, MD

Javier Olascoaga, MD

Adolfo Jiménez-Huete, MD

Mireya Fernández-

Fournier, MD

Lluis Ramió-Torrentà, MD

Alvaro Cobo-Calvo, MD

Montserrat Viñals, MD

Clara de Andrés, MD

Virginia Meca-Lallana, MD

Angeles Cervelló, MD

Carmen Calles, MD

Manuel Barón Rubio, MD

Cristina Ramo-Tello, MD

Ana Caminero, MD

Elvira Munteis, MD

Alfredo R. Antigüedad, MD

Yolanda Blanco, MD

Pablo Villoslada, MD

Xavier Montalban, MD

Francesc Graus, MD

Albert Saiz, MD

On behalf of the Spanish

NMO Study Group

Correspondence to

Dr. Saiz:

asaiz@clinic.ub.es

Supplemental data at Neurology.org/nn

\section{Neuromyelitis optica spectrum disorders}

\section{Comparison according to the phenotype and serostatus}

\section{OPEN}

\section{ABSTRACT}

Objective: To (1) determine the value of the recently proposed criteria of neuromyelitis optica (NMO) spectrum disorder (NMOSD) that unify patients with NMO and those with limited forms (NMO/LF) with aquaporin-4 immunoglobulin G (AQP4-lgG) antibodies; and (2) investigate the clinical significance of the serologic status in patients with $\mathrm{NMO}$.

Methods: This was a retrospective, multicenter study of 181 patients fulfilling the 2006 NMO criteria ( $n=127)$ or NMO/LF criteria with AQP4-IgG $(n=54)$. AQP4-lgG and myelin oligodendrocyte glycoprotein immunoglobulin G (MOG-lgG) antibodies were tested using cell-based assays.

Results: Patients were mainly white (86\%) and female (ratio 6.5:1) with median age at onset 39 years (range 10-77). Compared to patients with NMO and AQP4-IgG $(n=94)$, those with NMO/ LF presented more often with longitudinally extensive transverse myelitis (LETM) $(p<0.001)$, and had lower relapse rates $(p=0.015)$, but similar disability outcomes. Nonwhite ethnicity and optic neuritis presentation doubled the risk for developing NMO compared with white race $(p=0.008)$ or LETM presentation $(p=0.008$ ). Nonwhite race (hazard ratio [HR] 4.3, 95\% confidence interval [CI] 1.4-13.6) and older age at onset were associated with worse outcome (for every 10-year increase, HR 1.7, 95\% Cl 1.3-2.2). Patients with NMO and MOG-IgG $(n=9)$ had lower female: male ratio (0.8:1) and better disability outcome than AQP4-IgG-seropositive or doubleseronegative patients $(p<0.001)$.

Conclusions: In patients with AQP4-IgG, the similar outcomes regardless of the clinical phenotype support the unified term NMOSD; nonwhite ethnicity and older age at onset are associated with worse outcome. Double-seronegative and AQP4-IgG-seropositive NMO have a similar clinical outcome. The better prognosis of patients with MOG-lgG and NMO suggests that phenotypic and serologic classification is useful. Neurol Neuroimmunol Neuroinflamm 2016;3:e225; doi: $10.1212 / \mathrm{NXI} .0000000000000225$

\section{GLOSSARY}

AQP4-IgG = aquaporin-4 immunoglobulin G; $\mathbf{A R R}=$ annualized relapse rate; $\mathbf{C B A}=$ cell-based assay; $\mathbf{C l}=$ confidence interval; EDSS = Expanded Disability Status Scale; HR = hazard ratio; IQR = interquartile range; $\mathbf{L F}=$ limited forms; $\mathbf{M O G}=$ myelin oligodendrocyte glycoprotein; $\mathbf{M S}$ = multiple sclerosis; $\mathbf{N M O}=$ neuromyelitis optica; $\mathbf{N M O S D}$ = neuromyelitis optica spectrum disorders; $\mathbf{O R}=$ odds ratio.

Neuromyelitis optica (NMO) is an inflammatory CNS disorder that preferentially affects the optic nerve and spinal cord. The discovery of aquaporin-4 immunoglobulin G (AQP4-IgG) antibodies as a specific biomarker of NMO led in 2006 to the development of revised NMO diagnostic criteria that required both optic neuritis and myelitis and AQP4-IgG seropositivity as supportive criteria. ${ }^{1}$ In 2007 , the term NMO spectrum disorders (NMOSD) was introduced to include AQP4-IgG-seropositive patients with limited or inaugural forms (e.g., recurrent optic neuritis or longitudinally extensive transverse myelitis [LETM]), or with

Authors' affiliations are listed at the end of the article.

Coinvestigators are listed at Neurology.org/nn.

Funding information and disclosures are provided at the end of the article. Go to Neurology.org/nn for full disclosure forms. The Article Processing Charge was paid by the authors.

This is an open access article distributed under the terms of the Creative Commons Attribution-NonCommercial-NoDerivatives License 4.0 (CC BY-NC-ND), which permits downloading and sharing the work provided it is properly cited. The work cannot be changed in any way or used commercially. 
manifestations outside of the optic nerve and spinal cord (e.g., hiccups or nausea and vomiting) ${ }^{2}$

In 2015, an International Panel proposed new diagnostic criteria that unify patients with typical NMO and the more recently defined NMOSD under the term NMOSD. ${ }^{3}$ These criteria are based on the presence or absence of AQP4-IgG and on the assumption that patients with limited forms (NMO/LF) will develop typical NMO over time. ${ }^{2,3}$ However, in some patients the interval between the disease-defining events of optic neuritis and myelitis and conversion to NMO may be years or decades. ${ }^{2,4,5}$ Why some patients remain with limited forms for prolonged periods of time and if having a limited form impacts disability outcomes are unknown. Both the 2006 and the 2015 criteria allow the diagnosis of NMO or NMOSD, respectively, in patients without AQP4-IgG. ${ }^{6,7}$ Recent studies have shown that some of these patients have antibodies to myelin oligodendrocyte glycoprotein (MOG).$^{7-9}$ Moreover, studies suggest that the clinical outcome of patients with NMO and MOG-IgG is different from that of patients with NMO with AQP4-IgG or who are double seronegative. ${ }^{7,9}$

These questions are important because in the newly proposed criteria different clinical phenotypes such as $\mathrm{NMO}$ or $\mathrm{NMO} / \mathrm{LF}$ are no longer considered. To address this, we analyzed the demographic and clinical features of a Spanish cohort of patients with NMOSD with AQP4-IgG who had either the NMO phenotype (2006 criteria) or $\mathrm{NMO} / \mathrm{LF}$ to identify predictors of conversion to $\mathrm{NMO}$ and impact on disability outcomes. We also reviewed the clinical outcomes of patients with the NMO phenotype according to the presence or absence of AQP4-IgG or MOG-IgG.

METHODS Case selection and data collection. Clinical information and samples for this observational, retrospective, multicenter study were collected from 59 centers through the multiple sclerosis (MS) study group of the Spanish Society of Neurology and the Spanish MS Network (Red Española de Esclerosis Múltiple) from January 2013 to January 2015. Overall, 181 patients who at last follow-up fulfilled the $2006 \mathrm{NMO}$ criteria $(\mathrm{n}=127)$ or had NMO/LF associated with AQP4-IgG $(\mathrm{n}=54)$ were included. ${ }^{1,2}$ Epidemiologic data, including demographic, clinical, CSF (cell count, protein levels, and oligoclonal bands), MRI findings (brain MRI classified as normal and abnormal with or without the Paty or Barkhof criteria, ${ }^{10}$ and number and extension of spinal cord lesions), treatment, and outcome, were obtained from medical records and information collected from referring neurologists through a structured questionnaire designed for NMO. All serum samples were tested for AQP4IgG by immunohistochemistry and an in-house cell-based assay (CBA) with live HEK293 cells transfected with the aquaporin-4M23 isoform as reported. ${ }^{9,11}$ All but 5 samples from AQP4-IgGseropositive and 4 seronegative cases were tested for MOG-IgG using an in-house CBA with HEK293 cells transfected with the full-length MOG C-terminally fused to EGFP. ' The outcome reached at last follow-up was evaluated by the Expanded Disability Status Scale (EDSS) score. ${ }^{12}$ Severe visual disability was defined as sustained visual acuity $\leq 20 / 100$ with best correction possible during at least 6 months after an optic neuritis attack.

Standard protocol approvals, registrations, and patient consents. The study was approved by the Ethics Committee of the Hospital Clinic, and written consent was obtained for all participants. Samples were deposited in a registered biobank of the Institut dInvestigació Biomèdica August Pi i Sunyer (IDIBAPS), Barcelona, Spain.

Statistical methods. Characteristics were compared between patient groups using $\chi^{2}$ (or Fisher exact) tests for categorical data and Student $t$ test (or Wilcoxon rank sum) for continuous data. The Kaplan-Meier method was used to estimate the time to first and second recurrence, conversion to NMO phenotype, onset of chronic therapy, and to reach EDSS scores of 6 and 8. Survival curves were compared using log-rank tests and predictive factors for disability were assessed with Cox proportional hazards regression models. Disease characteristics along the disease course were compared using regression models with generalized estimating equations, logistic regression for binary data, and linear regression for continuous data, and adjusted by the follow-up time for each patient. Age, sex, ethnicity, type of syndrome at onset, and severity after the first event were included as predictive factors for conversion, and annualized relapse rate was added as predictive factor for disability. Chronic therapy was included as a time-dependent covariable in both analyses. Two-sided $p$ values $<0.05$ were considered statistically significant. Analyses were performed using SPSS version 19.0.

RESULTS Demographic, clinical, and serologic characteristics of the cohort. Clinical and demographic data of the 181 patients are summarized in table 1 . The median age at disease onset was 39 years (range 10-77 years) and the disease duration 6.4 years (0.2-50 years). Patients were mainly white (86\%), with a female:male ratio of $6.5: 1$. The frequency of optic neuritis or transverse myelitis presentation at onset was similar (38\% and $40 \%$, respectively); simultaneous or sequential ( $<1$ month from onset) optic and spinal attacks were infrequent (14\%). The clinical phenotype and disease course at the last followup is detailed in figure e-1 at Neurology.org/nn. All but 20 patients (11\%) had a relapsing course, and 150 $(83 \%)$ were on chronic therapy. In total, 148 patients (82\%) had AQP4-IgG, 9 (5\%) MOG-IgG, 2 (1\%) both, and $22(13 \%)$ were double seronegative. The 
Table 1 Demographic and disease characteristics of the cohort

Values (total $\mathrm{n}=181$ )

\section{Sex, female:male}

White ethnicity, n (\%)

Age at onset, $y$, median (range)

Coexisting autoimmune disease, $\mathrm{n}(\%)$

Onset attack type, $\mathrm{n}(\%)$

Optic neuritis

Myelitis

Simultaneous ${ }^{\mathrm{a}}$ optic neuritis + myelitis

Brainstem/brain

Follow-up duration, $y$, median (range)

Time to first relapse, mo, median $(95 \% \mathrm{Cl})$

Annualized relapse rate, mean \pm SD

Disability ${ }^{b, c}$

Outcome reached at last follow-up

Last EDSS score, median (range)

EDSS score $26.0, n(\%)$

EDSS score $\geq 8.0, \mathrm{n}$ (\%)

Visual acuity $\leq 20 / 100, n(\%)$

Patients who died, $\mathrm{n}$ (\%)
$3.5(0-9.0)$

$60(36)$

24 (15)

157:24 (ratio 6.5:1)

155 (86)

39 (10-77)

$40(22)$

69 (38)

$73(40)$

26 (14)

13 (7)

$6.4(0.2-50)$

$13(9-16)$

$1.0 \pm 1.3$

59 (42)

9 (5)

Abbreviations: $\mathrm{Cl}=$ confidence interval; EDSS = Expanded Disability Status Scale. a Simultaneous or sequential (less than 1 month from symptom onset) optic and spinal attack.

${ }^{b}$ For the EDSS outcome comparison (EDSS at last follow-up $\geq 6.0$ and $\geq 8.0$ ), only those patients who had at least one myelitis attack were considered $(n=165)$.

${ }^{c}$ For the visual outcome comparison (visual acuity at last follow-up $\leq 20 / 100$ ), only those patients who had at least one optic neuritis attack were considered $(n=140)$.

2 patients with both antibodies were excluded from the analyses of clinical-immunologic correlates.

Demographic and clinical differences between AQP4IgG-positive NMO patients and those who remained with NMO/LF. Examining the entire cohort of 148 AQP4-IgG patients showed those with NMO/LF presented more frequently with LETM at first $(p<$ $0.001)$ and second attack $(p=0.003)$ than those with NMO (table 2). The age at disease onset of the patients who presented with LETM and remained as NMO/LF was older than those who developed the NMO phenotype (median 47 vs 34 years, $p<0.001$ ).

We investigated which variables predicted conversion to NMO in this cohort. We found that nonwhite ethnicity doubled the risk as compared with white race (hazard ratio [HR] 2.2, 95\% confidence interval $[\mathrm{CI}], 1.2-3.9, p=0.008$ ); optic neuritis increased the risk 2-fold as compared with LETM presentation (HR 2.2, 95\% CI 1.2-3.9, $p=0.008$ ) and almost 4-fold as compared with brainstem attack (HR 3.9, 95\% CI 1.2-13.0, $p=0.024$ ). Age at disease onset only influenced the risk for developing $\mathrm{NMO}$ in those who presented with LETM, being

lower for older patients (30-40 years, HR 0.19, 95\% CI 0.07-0.55; $\geq 50$ years HR 0.054 , 95\% CI 0.01-0.27; compared with $<30$ years, $p<0.0001$ ). The 5-year conversion risk to NMO was $63.2 \%$ (95\% CI 48\%-78\%) for optic neuritis, $41.4 \%$ (95\% CI 26\%-57\%) for LETM, and 23\% (95\% CI 1\%-46\%) for brainstem syndrome (figure 1A).

Regarding disease severity, the mean annualized relapse rate (ARR) for the patients who remained with $\mathrm{NMO} / \mathrm{LF}$ was significantly lower (table 2); the mean number of relapses remained lower after adjusting for time of follow-up (3 vs 4.8, $p=0.018$ ). NMO/LF patients who remained monophasic had significantly shorter disease duration than those who relapsed (median 2 vs 5.5 years, $p<0.001$ ). Patients with $\mathrm{NMO} / \mathrm{LF}$ were treated earlier than those with NMO (median time from disease onset to therapy initiation 6.3 months [interquartile range (IQR) 3.7-18.6 months] vs 17.7 months [IQR 5.6-60 months], $p=0.014$ ), and had a better response; the mean (SD) ARR after treatment decreased to 0.3 (0.6) in NMO/LF and 0.7 (0.8) in NMO patients, $p=0.035$. Seventy of the 84 patients $(83 \%)$ who received chronic therapy were treated after fulfillment of NMO criteria (e-Results).

The EDSS score at last follow-up was significantly lower in those who remained $\mathrm{NMO} / \mathrm{LF}$ than in patients with NMO (table 2), but the difference was due to the lower EDSS score of the patients who remained as optic neuritis (median 2.0, range 1.0-4.0) or brainstem syndrome (median 1.0, range 0-2.0). When we compared the motor disability of patients with NMO with that of patients who remained with LETM, no significant differences were found in the proportion of patients who reached severe disability (EDSS score of 6.0). There was also no difference in the percentage of patients with severe visual disability (visual acuity $\leq 20 / 100$ ) when comparing patients with NMO with those who remained with optic neuritis (table 2). The same results were found after adjusting for time of follow-up (motor disability: odds ratio [OR] 0.69, 95\% CI 0.3-1.6, $p=0.385$; visual disability: OR 1.027, 95\% CI 0.29-3.65, $p=0.970)$.

Predictors for disability in patients with AQP4-IgG. In the analyses for the development of disability, we found that nonwhite ethnicity (HR 4.3, 95\% CI 1.4-13.6, $p<0.012$ ), older age at disease onset (HR 1.7 for every 10-year increase in age at onset; 95\% CI 1.3-2.2, $p<0.0001$ ) (figure 1B), and higher residual disability after first attack increased the risk of requiring a cane to walk (EDSS 6.0) (HR 1.3, 95\% CI 1.1-1.6, $p=0.017$ ). In the analysis for visual outcome, older age at disease onset (OR 1.9 for every 10 -year increase in age at onset; 95\% CI 1.22.9, $p=0.005)$, higher disability after the first attack 


\begin{tabular}{|c|c|c|c|}
\hline \multirow[t]{2}{*}{ Demographic and clinical feat } & \multirow[b]{2}{*}{ NMO $(n=94)^{a}$} & \multirow[b]{2}{*}{ NMO/LF ( $n=54)$} & \multirow[b]{2}{*}{ p Value } \\
\hline & & & \\
\hline Sex, female:male & $88: 6$ (ratio $14.7: 1$ ) & 50:4 (ratio 12.5:1) & 0.811 \\
\hline White ethnicity, $\mathrm{n}(\%)$ & $76(81)$ & $47(87)$ & 0.855 \\
\hline Age at onset, $y$, median (range) & $37(11-77)$ & $43(10-74)$ & 0.054 \\
\hline \multicolumn{4}{|l|}{ Onset attack type, n (\%) } \\
\hline Optic neuritis & $49(52)$ & $11(20)$ & \\
\hline Myelitis & $28(30)$ & $33(61)$ & $<0.001^{\mathrm{b}}$ \\
\hline Simultaneous ${ }^{c}$ optic neuritis + myelitis & $14(15)$ & 0 & \\
\hline Brainstem/brain & 3 (3) & $10(19)$ & \\
\hline EDSS score after first attack, median (range) & $3.0(0-8.0)$ & $3.3(0-7.0)$ & 0.315 \\
\hline Monophasic course, ${ }^{d}$ (\%) & $2(2)$ & $11(20)$ & $<0.001^{\mathrm{b}}$ \\
\hline Chronic treatment, n (\%) & $84(89)$ & $40(74)$ & $0.015^{\mathrm{b}}$ \\
\hline Follow-up, y, median (range) & $7.2(0.2-50)$ & $5.0(0.7-37)$ & $0.005^{\mathrm{b}}$ \\
\hline Annualized relapse rate, mean $\pm S D$ & $1.2 \pm 1.6$ & $0.7 \pm 0.5$ & $0.015^{b}$ \\
\hline \multicolumn{4}{|l|}{ Estimated $\%$ patients with relapses } \\
\hline Within 1 y of onset & 54 & 41 & 0.076 \\
\hline Within $2 y$ of onset & 67 & 53 & 0.076 \\
\hline Time to first relapse, mo, median $(95 \% \mathrm{Cl})$ & $12(9-15)$ & $22(10-34)$ & 0.076 \\
\hline \multicolumn{4}{|l|}{ Disability e,f } \\
\hline \multicolumn{4}{|l|}{ Outcome reached at last follow-up } \\
\hline Last EDSS score, median (range) & $4.5(1.0-9.0)$ & $2.5(0-8.0)$ & $<0.001^{\mathrm{b}}$ \\
\hline EDSS score $\geq 6.0, n$ (\%) & $38(40)$ & $14(37)$ & 1.00 \\
\hline EDSS score $\geq 8.0, n(\%)$ & $15(16)$ & $3(8)$ & 0.396 \\
\hline Visual acuity $\leq 20 / 100, n(\%)$ & $41(44)$ & $6(46)$ & 1.00 \\
\hline Patients who died, n (\%) & $7(7.5)$ & $1(1.9)$ & 0.147 \\
\hline
\end{tabular}

Abbreviations: AQP4-lgG = aquaporin-4 immunoglobulin G; Cl = confidence interval; EDSS = Expanded Disability Status Scale; LF = limited forms; NMO = neuromyelitis optica.

${ }^{a}$ Two patients with NMO with both AQP4-IgG and antibodies to myelin oligodendrocyte glycoprotein presented with a simultaneous optic and spinal attack and were excluded from the analyses.

b Significant.

c Simultaneous or sequential (less than 1 month from symptom onset) optic and spinal attack.

${ }^{\mathrm{d}}$ Monophasic course: patients without relapses after their initial attack.

${ }^{e}$ For the EDSS outcome comparison (EDSS at last follow-up $\geq 6.0$ and $\geq 8.0$ ), only those patients who remained as NMO/LF and who had at least one myelitis attack were considered $(n=38)$.

${ }^{f}$ For the visual outcome comparison (visual acuity at last follow-up $\leq 20 / 100$ ), only those patients who remained as NMO/ LF and who had at least one optic neuritis attack were considered $(n=13)$.

(OR 1.7, 95\% CI 1.1-2.6, $p=0.015$ ), and time of follow-up (by year, OR 1.2, 95\% CI 1.1-1.3, $p=$ 0.028 ) were associated with worse visual outcome. No significant effect was observed in association with ethnicity, initial syndrome, ARR, or chronic therapy.

Demographic and clinical differences among patients with NMO phenotype according to antibody status. Patients with NMO and AQP4-IgG had a significantly higher female:male ratio (14.7:1) than patients who were double seronegative $(1.4: 1)$ or with MOG-IgG (0.8:1), $p<0.001$ (table 3; table e-1). At last follow-up, patients with MOG-IgG had a lower median EDSS score (1.0, range 0-3.0) than those with AQP4-IgG (4.5, range 1.0-9.0) and those who were double seronegative $(5.0$, range $0-9.0$ ), $p<0.001$ (table 3). Due to the small sample size and the fact that none of the patients with MOG-IgG reached the EDSS score of 6.0, only a trend to lower risk of motor disability was observed in comparison with AQP4-IgG and double seronegative patients (log-rank test, $p=0.089$ ) (figure 1C). There were no differences among the 3 groups in severity of disease at onset, time to first relapse, ARR, and frequency of relapses within 1 or 2 years of onset (table 3). The median delay to start therapy was not significantly different among the groups (around 0.51.5 years), and the comparative analyses of the effect of 
Figure 1 Kaplan-Meier estimation of time to neuromyelitis optica (NMO) conversion and development of motor disability

A. Time to NMO conversion by onset attack type

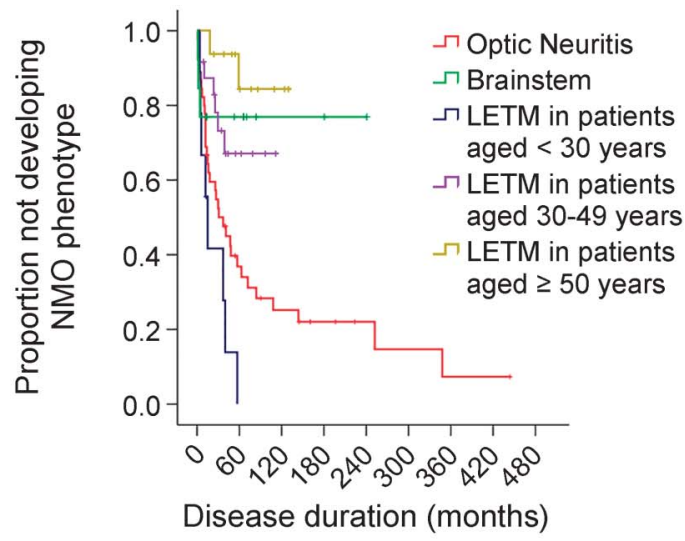

B. Time to EDSS 6.0 by age at onset

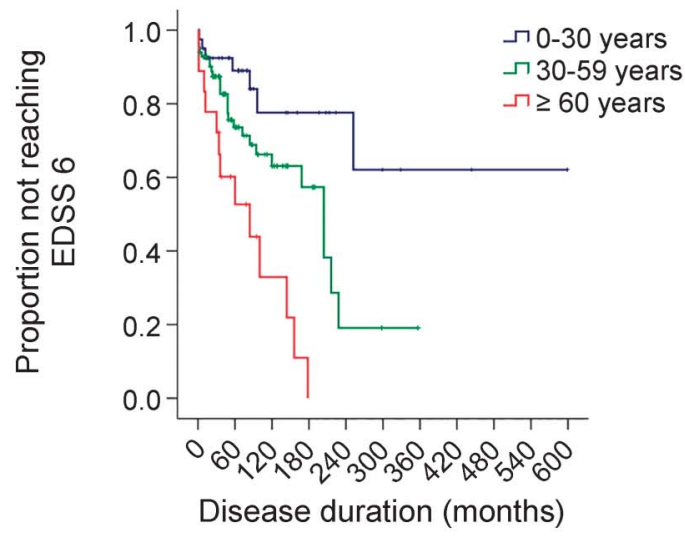

C. Time to EDSS 6.0 by serostatus

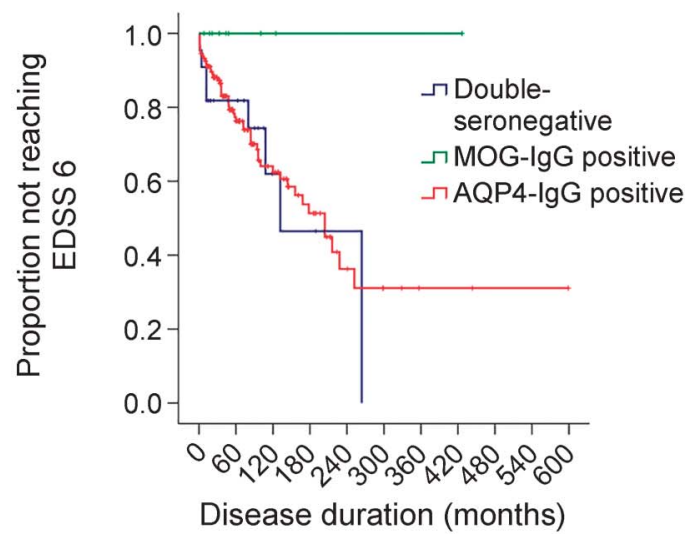

(A) Months from onset to develop NMO according to the onset attack type: patients with optic neuritis, and those with longitudinally extensive transverse myelitis (LETM) aged $<30$ years, converted earlier than patients with LETM aged $>30$ years or with brainstem syndrome $(p<0.001)$. (B) Months from onset to use a cane (Expanded Disability Status Scale [EDSS] score 6.0) by age at disease onset: older patients were significantly more likely than younger patients to develop motor disability over time $(p<0.001)$. therapy on relapse rate did not reveal significant differences; after therapy, the mean (SD) ARR for AQP4-IgG patients decreased from 3.6 (5.5) to 0.7 (0.8), for double seronegative from 3.2 (3.7) to 0.6 (1.1), and for MOG-IgG from 5.2 (3.9) to $0.1(0.2)$ ( $p=0.400)$ (e-Results).

DISCUSSION This study of a large cohort of NMO patients identified by uniform criteria and sensitive assays for AQP4-IgG and MOG-IgG detection provides several important observations: (1) the median time of conversion to $\mathrm{NMO}$ in patients with AQP4-IgG who develop the NMO phenotype is 1 year; however, there is a subgroup of patients with AQP4-IgG who do not convert to NMO after a median follow-up of 5 years; (2) presentation with optic neuritis and nonwhite ethnicity are predictors of NMO conversion; (3) the final motor or visual disability outcome of patients with AQP4-IgG who do and do not convert to NMO is the same; (4) older age and nonwhite ethnicity are predictors of worse disability outcome irrespective of syndrome; (5) patients with NMO and AQP4-IgG and those who are double seronegative have similar clinical profiles in terms of relapses and disability; and (6) patients with NMO and MOG-IgG have better outcomes compared to patients with NMO and AQP4-IgG or who are double seronegative.

The 2015 proposed diagnostic criteria unifying the traditional NMO and modern NMOSD definitions for patients with AQP4-IgG was predicated on an absence of differences in clinical behavior, immunopathogenesis, and treatment of these patients. ${ }^{3}$ Our results support this aspect of the criteria as we found that all AQP4-IgG-seropositive patients have similar motor or visual disability outcomes.

We observed that presentation as optic neuritis increased the risk of developing NMO compared with presentation as LETM, but this was only significant for those patients with LETM aged $\geq 30$ years. The higher risk of developing NMO in patients who present with optic neuritis was also observed in a previous study of patients with AQP4-IgG-seropositive NMOSD, although the influence of age was not noticed. ${ }^{5}$ Similarly, these authors also found a greater likelihood of developing NMO in Afro-Caribbean

(C) Years from onset to use a cane by antibody status in NMO patients: at 5 years after onset, $26 \%$ of aquaporin4 immunoglobulin G (AQP4-lgG)-positive patients, 19\% of double-seronegative patients, and none of the myelin oligodendrocyte glycoprotein (MOG)-lgG-positive patients were expected to need a cane to walk (EDSS score 6.0) ( $p=$ 0.089). EDSS score $6.0=$ intermittent or unilateral assistance required to walk 100 meters with or without resting. $\mathrm{ON}=$ optic neuritis. 


\begin{tabular}{|c|c|c|c|c|c|}
\hline \multirow[t]{2}{*}{ Table 3} & \multicolumn{5}{|c|}{$\begin{array}{l}\text { Comparison of demographic and clinical characteristics of patients with NMO (fulfilling the } 2006 \text { NMO criteria) according to antibody } \\
\text { status }\end{array}$} \\
\hline & & $\begin{array}{l}\text { AQP4-IgG-positive } \\
(\mathrm{n}=94)^{\mathrm{a}}\end{array}$ & $\begin{array}{l}\text { Double seronegative } \\
(\mathrm{n}=22)\end{array}$ & $\begin{array}{l}\text { MOG-IgG-positive } \\
(\mathrm{n}=9)^{\mathrm{a}}\end{array}$ & $p$ Value \\
\hline \multicolumn{2}{|c|}{ Sex, female:male } & $88: 6$ (ratio 14.7:1) & 13:9 (ratio 1.4:1) & $4: 5$ (ratio 0.8:1) & $<0.001^{\mathrm{b}}$ \\
\hline \multicolumn{2}{|c|}{ White ethnicity, n (\%) } & $76(81)$ & $21(96)$ & $9(100)$ & 0.773 \\
\hline \multicolumn{2}{|c|}{ Coexisting autoimmune diseases, $n(\%)$} & $18(19)$ & 7 (32) & $1(11)$ & 0.318 \\
\hline \multicolumn{6}{|c|}{ Onset attack type, n (\%) } \\
\hline \multicolumn{2}{|c|}{ Optic neuritis } & $49(52)$ & 7 (32) & $2(22)$ & \\
\hline \multicolumn{2}{|c|}{ Myelitis } & $28(30)$ & $9(41)$ & 3 (33) & 0.166 \\
\hline \multicolumn{2}{|c|}{ Simultaneous ${ }^{c}$ optic neuritis + myelitis } & $14(15)$ & $6(27)$ & $4(45)$ & \\
\hline \multicolumn{2}{|c|}{ Monophasic course, ${ }^{d}$ (\%) } & 2 (2) & $3(14)$ & $2(22)$ & $0.008^{b}$ \\
\hline \multicolumn{2}{|c|}{ Chronic treatment, $\mathrm{n}(\%)$} & $84(89)$ & $18(82)$ & $6(67)$ & 0.130 \\
\hline \multicolumn{2}{|c|}{ Follow-up, y, median (range) } & $7.2(0.2-50)$ & $6.4(1-36)$ & $3.7(0.7-36)$ & 0.232 \\
\hline \multicolumn{2}{|c|}{ Median time to develop $\mathrm{NMO}^{\mathrm{e}} \mathrm{mo}$, interquartile range } & $12(3-37)$ & $6.5(1-18)$ & $6(1-44)$ & 0.521 \\
\hline \multicolumn{2}{|c|}{ No. of relapses, median (range) } & $4(1-27)$ & $2(1-11)$ & $3(1-7)$ & 0.129 \\
\hline \multicolumn{6}{|c|}{ Estimated $\%$ patients with relapses } \\
\hline \multicolumn{2}{|c|}{ Within 1 y of onset } & 54 & 47 & 56 & 0.820 \\
\hline \multicolumn{2}{|c|}{ Within $2 \mathrm{y}$ of onset } & 69 & 66 & 56 & 0.820 \\
\hline \multicolumn{2}{|c|}{ Time to first relapse, mo, median $(95 \% \mathrm{Cl})$} & $12(9-15)$ & $13(8-18)$ & $7(4-11)$ & 0.820 \\
\hline \multicolumn{6}{|c|}{ Outcome reached at last follow-up } \\
\hline \multicolumn{2}{|c|}{ Visual acuity $\leq 20 / 100, \%$} & $41(44)$ & $11(50)$ & $1(11)$ & 0.112 \\
\hline \multicolumn{6}{|c|}{$\begin{array}{l}\text { Kaplan-Meier estimated \% of patients expected } \\
\text { to reach disability outcome at } 5 \text { y after onset }\end{array}$} \\
\hline \multicolumn{2}{|c|}{ EDSS $\geq 6.0$} & 26 & 19 & 0 & 0.089 \\
\hline \multicolumn{2}{|c|}{ EDSS $\geq 8.0$} & 10 & 12 & 0 & 0.196 \\
\hline \multicolumn{2}{|c|}{ Patients who died, n (\%) } & $7(7.5)$ & $1(4.5)$ & 0 & 0.658 \\
\hline
\end{tabular}

Abbreviations: AQP4-IgG = aquaporin-4 immunoglobulin G; Cl = confidence interval; EDSS = Expanded Disability Status Scale; MOG = myelin oligodendrocyte glycoprotein; NMO = neuromyelitis optica.

${ }^{a}$ Two patients with NMO with both AQP4-IgG and MOG-IgG presented with a simultaneous optic and spinal attack and were excluded from the analyses.

bignificant.

${ }^{\mathrm{c}}$ Simultaneous or sequential (less than 1 month from symptom onset) optic and spinal attack.

${ }^{\mathrm{d}}$ Monophasic course: patients without relapses after their initial attack.

${ }^{e}$ Fulfillment of the 2006 NMO criteria. ${ }^{1}$

patients than Caucasians, ${ }^{5}$ supporting a contribution of genetic factors to the course of the disease.

Our results confirm previous observations that patients with AQP4-IgG who remain as NMO/LF present more frequently with LETM and are older than those who convert to NMO., 5,134 The cause of the lower risk for patients with LETM to develop NMO is not clear. In our cohort of AQP4-IgG-positive patients, those with LETM were treated earlier than those with optic neuritis, and immunotherapy could have prevented the development of NMO. However, early treatment would not explain the differences observed between younger ( $<30$ years) and older $(\geq 50$ years) patients with LETM. Moreover, in patients with NMO/LF, the time to therapy initiation was not significantly different between patients who presented with optic neuritis or LETM.

There is widespread acceptance that attack prevention with immunosuppressant therapies is effective in reducing NMO relapses, and our study confirms a significant reduction in the relapse rate in both 
AQP4-IgG NMO and NMO/LF groups. The fact that patients with AQP4-IgG NMO/LF who remained monophasic had significantly shorter disease duration than those who relapsed supports the need of a follow-up of at least 5 years to qualify as a monophasic disease course, as suggested by the 2015 criteria. ${ }^{3}$ The contribution, however, of early therapeutic intervention to modulate the natural history of $\mathrm{NMO} / \mathrm{LF}$ remains unclear and will only be ascertained with prospective studies in which clinical phenotype classification will be relevant. Until these studies are done, as disability outcome is similar for patients with AQP4-IgG who do and do not convert to NMO, as well as for patients who are double seronegative, early preventive therapy is warranted in these patients.

The 2015 criteria allow the diagnosis of NMOSD in patients without AQP4-IgG. ${ }^{6,7}$ This is a matter of debate. Previous studies showed some clinical differences between AQP4-IgG-positive and -negative patients, ${ }^{15}$ including a lower female predominance in those without AQP4-IgG, ${ }^{4,6}$ similar to the findings in the current study (1.4 vs 14.7 ). The phenotypic variability between AQP4-IgG-positive and -negative patients may be partially explained by the presence of MOG-IgG in a subgroup of the AQP4-IgG-seronegative patients. In the current study, we identified MOG-IgG in $29 \%$ of patients who would have been classified as seronegative. Compared to AQP4-IgGseropositive or double-seronegative patients, those with MOG-IgG showed a male predominance, more often presented with simultaneous or sequential $(<1$ month from onset) optic and spinal attacks, and more commonly had a monophasic disease course. Additionally, these patients had better outcomes even though the severity of disability during the initial attack and relapse rates were similar to the AQP4IgG-seropositive or double-seronegative patients. These data and prior studies confirm the distinct prognosis for patients with MOG-IgG, ${ }^{7,9,16}$ and support the importance of testing for MOG-IgG. Moreover, our study confirms that double-positive cases are very rare. The 2 double-positive patients presented with simultaneous optic neuritis and LETM, and had a typical relapsing course. This is in contrast with a recently reported case that presented with an acute demyelinating encephalomyelitis and developed a fulminant clinical course. ${ }^{17}$

Even with the addition of MOG-IgG testing, 19\% of patients in our study remained seronegative. This group of patients had similar clinical features and disability outcome to AQP4-IgG-seropositive patients, consistent with a recent report of the Mayo Clinic. ${ }^{6}$ For example, the percentage of AQP4-IgG-seropositive patients who relapsed within the first $(51 \%)$ or second $(62 \%)$ year after onset, and the estimated median time to require a cane ( 15 years), was similar. ${ }^{6}$ The challenge will be to determine if the seronegativity of this group is due to a lack of sensitivity of current antibody assays or the presence of uncharacterized immune responses. Additionally, it is likely that this is not a clinically homogenous group, highlighting the importance of collecting detailed phenotypic and epidemiologic data.

Our study has limitations related to its retrospective nature, and the ascertainment bias related to selection of patients who fulfilled the 2006 and 2007 definitions that were more restrictive than the recently proposed criteria. However, our findings in this large cohort of patients support the unified definition of NMOSD for seropositive AQP4-IgG patients, and provide novel serologic and clinical predictors of outcome.

\section{AUTHOR AFFILIATIONS}

From the Center of Neuroimmunology (M.S., T.A., N.S.-V., Y.B., P.V., F.G., A.S.), Service of Neurology, Hospital Clínic and Institut dInvestigació Biomèdica August Pi i Sunyer (IDIBAPS), Universitat de Barcelona; Neuroimmunology Unit (T.A.), Department of Child Neurology, Sant Joan de Déu Hospital, University of Barcelona, Esplugues del Llobregat; Servei de Neurologia-Neuroimmunologia (G.A., X.M.), Centre dEsclerosi Múltiple de Catalunya (CEM-Cat), Vall dHebron Institut de Recerca, Hospital Universitari Vall dHebron, Universitat Autònoma de Barcelona; Multiple Sclerosis Unit (J.E.M.-L.), Department of Neurology, Hospital Clínico Universitario Virgen de la Arrixaca, Cátedra de Neuroinmunología Clínica y Esclerosis Múltiple, UCAM Universidad Católica San Antonio de Murcia; Department of Neurology (C.O.-G.), University Hospital San Carlos, IdISSC, Madrid; Department of Neurology (M.M.), Hospital Universitario de Cruces, Baracaldo; Department of Neurology (A.A.d.A.), Alava University Hospital, Txagorritxu, Vitoria-Gasteiz; Department of Neurology (Y.A.), University Hospital of Getafe; Department of Neurology (B.C.), Hospital Universitari La Fe, Valencia; Hospital Universitario de Donostia (J.O.), San Sebastián; Hospital Ruber Internacional (A.J.-H.), Madrid; Clinical Neuroimmunology and Multiple Sclerosis Unit (M.F.-F.), La Paz University Hospital, Madrid; Neuroimmunology and Multiple Sclerosis Unit (L.R.-T.), Department of Neurology, Dr. Josep Trueta University Hospital, IDIBGI, Girona; Multiple Sclerosis Unit (A.C.-C.), Neurology Department, Hospital Universitari de Bellvitge, LHospitalet de Llobregat; Neurology Department (M.V.), Reina Sofia Hospital, University of Cordoba; Department of Neurology (C.d.A.), Hospital General Universitario Gregorio Marañón, Madrid; Department of Neurology (V.M.-L.), Hospital Universitario de la Princesa, Madrid; Department of Neurology (A.C.), Hospital General Universitario de Valencia; Department of Neurology (C.C.), Hospital Universitari Son Espases, Palma de Mallorca; Department of Neurology (M.B.R.), Hospital Universitario Fundación Alcorcón, Madrid; Neurology Department (C.R.-T.), Hospital Universitario Germans Trias i Pujol, Badalona; Department of Neurology (A.C.), Hospital Nuestra Señora de Sonsoles, Avila; Department of Neurology (E.M.), Hospital del Mar, IMIM (Institut Hospital del Mar d'Investigacions Mèdiques), Barcelona; and Service of Neurology (A.R.A.), Hospital Universitario de Basurto, Bilbao, Spain.

\section{AUTHOR CONTRIBUTIONS}

Design/conceptualization of the study: M.S., F.G., A.S. Analysis/interpretation of the data, drafting and revising the manuscript: M.S., T.A., N.S.-V., Y.B., F.G., A.S. Statistical analysis: M.S., Y.B. Acquisition of data and revising the manuscript for intellectual content: G.A., J.E.M.-L., C.O.-G., M.M., A.A.d.A., Y.A., B.C., J.O., A.J.-H., M.F.-F., L.R.-T., A.C.-C., M.V., C.d.A., V.M.-L., A.C., C.C., M.B.R., C.R.-T., A.C., E.M., A.R.A., P.V., X.M. All authors have given final approval of the manuscript. 


\section{ACKNOWLEDGMENT}

The authors thank Dr. Myrna Rosenfeld for critical review of the manuscript and Eva Caballero and Mercè Alba for technical assistance.

\section{STUDY FUNDING}

This study was supported in part by Red Española de Esclerosis Múltiple (REEM) Instituto de Salud Carlos III, Spain (RD07/0060/01, P.V.; RD12/0032/0002, A.S.; Marató de TV3 [20141830], F.G.) and Instituto de Salud Carlos III, Spain (CM14/00081; T.A.).

\section{DISCLOSURE}

M. Sepúlveda reports no disclosures. T. Armangué is an editorial advisory board member for Pediatria Catalana and receives research support from Instituto Carlos III (Spain), Asociacion Española de Pediatria, and Fundacio Marato TV3. N. Sola-Valls reports no disclosures. Georgina Arrambide has consulted for Biogen-Idec. J.E. Meca-Lallana has consulted for Biogen-Idec, Sanofi-Aventis, and Teva Pharmaceuticals and received speaker honoraria from Almirall, Merck-Serono, Biogen-Idec, Sanofi-Aventis, Teva Pharmaceuticals, and Novartis. C. Oreja-Guevara has consulted for Biogen Idec, Genzyme, Merck-Serono, and Novartis and received speaker honoraria from Biogen Idec, Genzyme, MerckSerono, and Novartis. M. Mendibe, A. Alvarez de Arcaya, and Y. Alandro report no disclosures. B. Casanova served on the scientific advisory board for Roche, Biogen-Idec, Genzyme, Teva, Novartis, and Merck-Serono; has consulted for Roche, Biogen-Idec, Genzyme, Teva, Novartis, and Merck-Serono; and received research support from Biogen-Idec, Genzyme, and Teva. J. Olascoaga served on the scientific advisory board for Biogen Idec, Genzyme, Novartis, Roche, and Teva; received travel funding and/or speaker honoraria from Biogen-Idec, Bayer-Schering, Genzyme, Merck-Serono, Novartis, and Teva; and received research support from Biogen Idec, Merck Serono, Novartis, and Teva. A Jiménez-Huete and M. Fernández-Fournier report no disclosures. L. Ramió-Torrentà received travel funding and/or speaker honoraria from Biogen Idec, Bayer, Merck-Serono, Teva Pharmaceuticals Industries Ltd., Novartis, Genzyme, and Almirall. A. Cobo-Calvo, M. Viñals, and C. de Andrés report no disclosures. V. Meca-Lallana served on the scientific advisory board for Genzyme and Roche and received travel funding and/ or speaker honoraria from Genzyme, Sanofi-Aventis, Merck-Serono, Teva Pharmaceuticals Industries Ltd., Novartis, Biogen Idec, and Terumo. A Cervelló reports no disclosures. C. Calles received speaker honoraria from Biogen Idec, Bayer, Merck-Serono, Genzyme, Teva Pharmaceuticals Inc, and Novartis, and received research support from Biogen Idec, Bayer, Merck-Serono, Genzyme, Teva Pharmaceutical Industries Ltd., and Novartis. M.B. Rubio, C. Ramo-Tello, and A. Caminero report no disclosures. E. Munteis received speaker honoraria from Biogen Idec, Teva Pharmaceutical Industries Ltd., Novartis, Almirall, and Genzyme, and received research support from Biogen and Merck-Serono. A.R. Antigüedad and Y. Blanco report no disclosures. P. Villoslada served on the scientific advisory board for Roche, Novartis, Bionure, and Genzyme; received travel funding and/or speaker honoraria from Novartis, Roche, and Genzyme; is an academic editor for PloSONE; is on the editorial board for Neurology \& Therapy, Curr Treat Options Neurol, and Multiple Sclerosis and Demyelinating Disorders; holds patents for methylthioadenosine for the treatment of MS, agnostic neurotrophic compounds for the treatment of brain diseases, gene signature pattern as a biomarker for MS, and algorithm for quantifying fractal dimension in brain MTI; and received research support from Novartis, Roche, Genzyme, Instituto de Salud Carlos III, European Commission, National MS Society, Fundación Maraton TV3, Bionure Inc., and Spire Bioventures Mintlabs. X. Montalban served on the scientific advisory board for Bayer-Schering, Biogen-Idec, EMD Merck-Serono, Genentech, Genzyme, Novartis, Sanofi-Aventis, Teva Pharmaceuticals, and Almirall; received travel funding from Novartis, Teva Pharmaceutical, EMD Merck-Serono, Biogen-Idec, Bayer-Schering Pharma, Almirall, Genzyme, and Sanofi-Aventis; is on the editorial board for Multiple Sclerosis Journal, Journal of Neurology, The International MS Journal, Revista de Neurologia, and Therapeutic Advances in Neurological Disorders; has consulted for Bayer-Schering Pharma, Biogen-Idec, Genzyme, Merck, Novartis, Sanofi-Aventis, and Teva Pharmaceutical; and received research support from Multiple Sclerosis Foundation of Barcelona. F. Graus is on the editorial board for Lancet Neurology, receives royalties from Euroimmun, and holds a patent for use of IgLON5 as a diagnostic test. A. Saiz is on the scientific advisory board for Biogen Idec and Merck Serono; received travel funding and/or speaker honoraria from Bayer-Schering, MerckSerono, Biogen Idec, Sanofi-Aventis, Teva Pharmaceutical Industries, and Novartis; and has consulted for Bayer-Schering, Merck Serono, Biogen Idec, Sanofi-Aventis, Teva Pharmaceutical, and Novartis. Go to Neurology.org/nn for full disclosure forms.

Received January 25, 2016. Accepted in final form February 24, 2016.

\section{REFERENCES}

1. Wingerchuk DM, Lennon VA, Pittock SJ, Lucchinetti CF, Weinshenker BG. Revised diagnostic criteria for neuromyelitis optica. Neurology 2006;66: 1485-1489.

2. Wingerchuk DM, Lennon VA, Lucchinetti CF, Pittock SJ, Weinshenker BG. The spectrum of neuromyelitis optica. Lancet Neurol 2007;6:805-815.

3. Wingerchuk DM, Banwell B, Bennett JL, et al. International consensus diagnostic criteria for neuromyelitis optica spectrum disorders. Neurology 2015;85:177-179.

4. Jarius S, Ruprecht K, Wildemann B, et al. Contrasting disease patterns in seropositive and seronegative neuromyelitis optica: a multicentre study of 175 patients. J Neuroinflammation 2012;9:14.

5. Kitley J, Leite MI, Nakashima I, et al. Prognostic factors and disease course in aquaporin- 4 antibody-positive patients with neuromyelitis optica spectrum disorder from the United Kingdom and Japan. Brain 2012;135: 1834-1849.

6. Jiao Y, Fryer JP, Lennon VA, et al. Updated estimate of AQP4-IgG serostatus and disability outcome in neuromyelitis optica. Neurology 2013;81:1197-1204.

7. Sato DK, Callegaro D, Lana-Peixoto MA, et al. Distinction between MOG antibody-positive and AQP4 antibodypositive NMO spectrum disorders. Neurology 2014;82: 474-481.

8. Kitley J, Waters P, Woodhall M, et al. Neuromyelitis optica spectrum disorders with aquaporin-4 and myelinoligodendrocyte glycoprotein antibodies: a comparative study. JAMA Neurol 2014;71:276-283.

9. Höftberger R, Sepulveda M, Armangue T, et al. Antibodies to MOG and AQP4 in adults with neuromyelitis optica and suspected limited forms of the disease. Mult Scler 2015;21:866-874.

10. Barkhof F, Filippi M, Miller D, et al. Comparison of MRI criteria at first presentation to predict conversion to clinically definite multiple sclerosis. Brain 1997;120: 2059-2069.

11. Höftberger R, Sabater L, Marignier R, et al. An optimized immunohistochemistry technique improves NMO-IgG detection: study comparison with cell-based assays. PLoS One 2013;8:e79083.

12. Kurtzke JF. Rating neurologic impairment in multiple sclerosis: an expanded disability status scale (EDSS). Neurology 1983;33:1444-1452.

13. Nagaihi A, Takagi M, Umemura A, et al. Clinical features of neuromyelitis optica in a large Japanese cohort: comparison between phenotypes. J Neurol Neurosurg Psychiatry 2011;82:1360-1364.

14. Jiao Y, Fryer JP, Lennon VA, et al. Aquaporin $4 \mathrm{IgG}$ serostatus and outcome in recurrent longitudinally extensive transverse myelitis. JAMA Neurol 2014;71: $48-54$. 
15. Marignier R, Bernard-Valnet R, Giraudon P, et al. Aquaporin-4 antibody-negative neuromyelitis optica: distinct assay sensitivity-dependent entity. Neurology 2013;80:2194-2200.

16. Cobo-Calvo A, Sepúlveda M, Bernard-Valnet R, et al. Antibodies to myelin oligodendrocyte glycoprotein in aquaporin 4 antibody seronegative longitudinally extensive transverse myelitis: clinical and prognostic implications. Mult Scler 2016;22:312-319.

17. Di Pauli F, Höftberger R, Reindl M, et al. Fulminant demyelinating encephalomyelitis: insights from antibody studies and neuropathology. Neurol Neuroimmunol Neuroinflamm 2015;2:e175. 


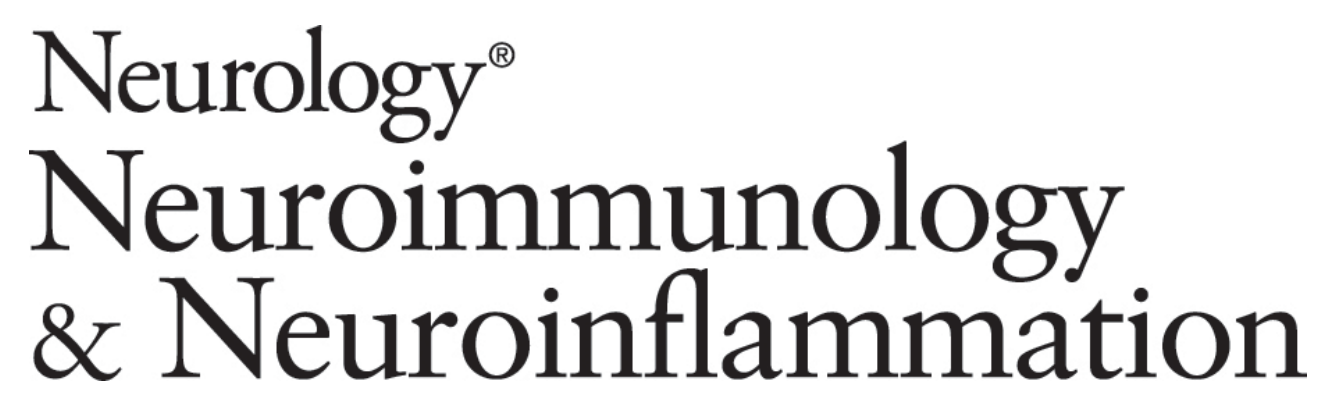

Neuromyelitis optica spectrum disorders: Comparison according to the phenotype and serostatus

Maria Sepúlveda, Thaís Armangué, Nuria Sola-Valls, et al.

Neurol Neuroimmunol Neuroinflamm 2016;3;

DOI 10.1212/NXI.0000000000000225

This information is current as of April 14, 2016

Neurol Neuroimmunol Neuroinflamm is an official journal of the American Academy of Neurology.

Published since April 2014, it is an open-access, online-only, continuous publication journal. Copyright $\odot$ 2016 American Academy of Neurology. All rights reserved. Online ISSN: 2332-7812.

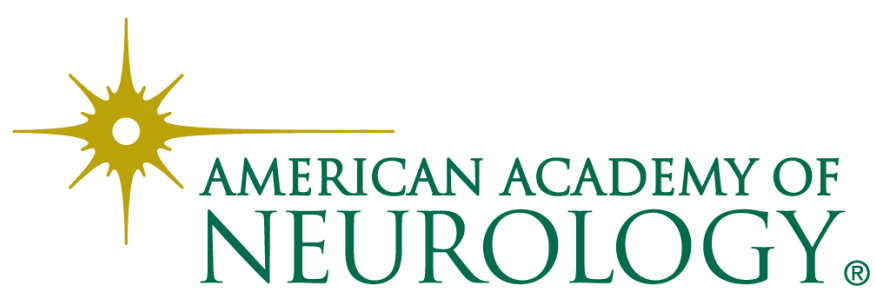




\section{Updated Information \& Services}

\section{Supplementary Material}

\section{References}

Citations

Subspecialty Collections

Permissions \& Licensing

\section{Reprints}

including high resolution figures, can be found at: http://nn.neurology.org/content/3/3/e225.full.html

Supplementary material can be found at: http://nn.neurology.org/content/suppl/2016/04/14/3.3.e225.DC1 http://nn.neurology.org/content/suppl/2016/04/14/3.3.e225.DC2

This article cites 17 articles, 1 of which you can access for free at: http://nn.neurology.org/content/3/3/e225.full.html\#\#ref-list-1

This article has been cited by 4 HighWire-hosted articles: http://nn.neurology.org/content/3/3/e225.full.html\#\#otherarticles

This article, along with others on similar topics, appears in the following collection(s):

\section{All Demyelinating disease (CNS)}

http://nn.neurology.org//cgi/collection/all_demyelinating_disease_cns Autoimmune diseases

http://nn.neurology.org//cgi/collection/autoimmune_diseases

Devic's syndrome

http://nn.neurology.org//cgi/collection/devics_syndrome

Optic neuritis; see Neuro-ophthalmology/Optic Nerve

http://nn.neurology.org//cgi/collection/optic_neuritis

Transverse myelitis

http://nn.neurology.org//cgi/collection/transverse_myelitis

Information about reproducing this article in parts (figures,tables) or in its entirety can be found online at:

http://nn.neurology.org/misc/about.xhtml\#permissions

Information about ordering reprints can be found online: http://nn.neurology.org/misc/addir.xhtml\#reprintsus

Neurol Neuroimmunol Neuroinflamm is an official journal of the American Academy of Neurology.

Published since April 2014, it is an open-access, online-only, continuous publication journal. Copyright $\odot$ 2016 American Academy of Neurology. All rights reserved. Online ISSN: 2332-7812.

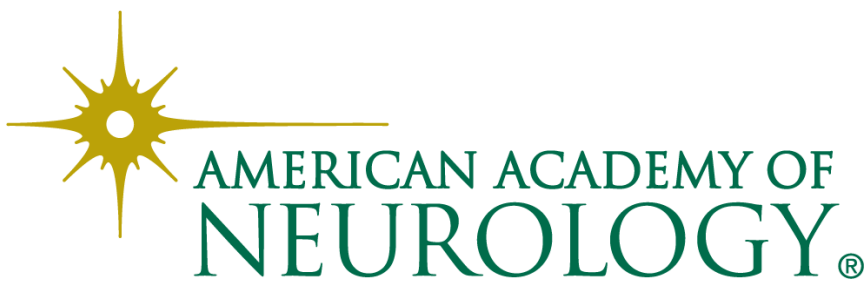

